East African Medical Journal Vol. 85 No. 11 November 2008

ADMISSION CHARACTERISTICS, DIAGNOSES AND OUTCOMES OF HIV -INFECTED PATIENTS REGISTERED IN AN AMBULATORY HIV-CARE PROGRAMME IN WESTERN KENYA

A. M. Siika, MMed, Department of Medicine, Moi University School of Medicine, P.O. Box 4604 - 30100, Eldoret, Kenya, Academic Model for Prevention and Treatment of HIV, Eldoret, Kenya, Indiana University School of Medicine, Indianapolis, Indiana, USA. P. O. Ayuo, MMed, Department of Medicine, Moi University School of Medicine, Eldoret, Kenya, Academic Model for Prevention and Treatment of HIV, Eldoret, Kenya, A. W. Mwangi, MSc, Academic Model for Prevention and Treatment of HIV, Eldoret. Kenya, Warren Alpert School of Medicine at Brown University, Providence, Rhode Island, USA, J. E. Sidle, K. Wools-Kaloustian, MD, Department of Medicine, Moi University School of Medicine, P.O. Box 4604 - 30100, Eldoret, Kenya, Academic Model for Prevention and Treatment of HIV, Eldoret, Kenya, Indiana University School of Medicine, Indianapolis, Indiana, USA, S. N. Kimaiyo, MMed, Department of Medicine, Moi University School of Medicine, Eldoret, Kenya, Academic Model for Prevention and Treatment of HIV, Eldoret, Kenya, and William, M.Tierney, MD, Academic Model for Prevention and Treatment of HIV, Eldoret, Kenya, Indiana University School of Medicine, Indianapolis, Indiana, USA, Regenstrief Institute, Inc, Indianapolis, Indiana, USA

Request for reprints to: Dr. A. M. Siika, Department of Medicine, Moi University School of Medicine, P. O. Box 460630100, Eldoret, Kenya

\title{
ADMISSION CHARACTERISTICS, DIAGNOSES AND OUTCOMES OF HIV-INFECTED PATIENTS REGISTERED IN AN AMBULATORY HIV-CARE PROGRAMME IN WESTERN KENYA
}

\author{
A. M. SIIKA, P. O. AYUO, A. W. MWANGI J. E. SIDLE, K. WOOLS-KALOUSTIAN, \\ S. N. KIMAIYO, and W. M. TIERNEY
}

\begin{abstract}
Objective: To determine admissions diagnosis and outcomes of HIV-infected patients attending AMPATH ambulatory HIV-care clinics.

Design: Prospective cohort study.

Setting: Academic Model for Prevention and Treatment of HIV/ AIDS (AMPATH) ambulatory HIV-care clinic in western Kenya.

Results: Between January 2005 and December 2006, 495 HIV-infected patients enrolled in AMPATH were admitted. Median age at admission was 38 years (range: 19-74), 62\% females, 375 (76\%) initiated cART a median 56 days (range: 1- 1288) before admission. Majority $(53 \%)$ had pre-admission CD4 counts $<100$ cells $/ \mathrm{ml}$ and $23 \%$ had counts $>\mathbf{2 0 0}$ cells $/ \mathrm{ml}$. Common admissions diagnoses were: tuberculosis (27\%); pneumonia $(15 \%)$; meningitis $(11 \%)$; diarrhoea $(11 \%)$; malaria $(6 \%)$; severe anaemia $(4 \%)$; and toxoplasmosis (3\%). Deaths occurred in $147(30 \%)$ patients who enrolled at AMPATH a median 44 days (range: 1 - 711) before admission and died a median 41 days (range: $1-713)$ after initiating cART. Tuberculosis (27\%) and meningitis (14\%) were the most common diagnoses in the deceased. Median admission duration was six days (range: 1 - 30) for deceased patients and eight days (range: $I$ - 44) for survivors $(P=0.0024)$. Deceased patients enrolled in AMPATH or initiated cART more recently, had lower CD4 counts and were more frequently lost to follow-up than survivors $(P<0.05$ for each comparison). Initiation of cART before admission and clinic appointment adherence were independent predictors of survival.

Conclusion: Although high mortality rate is seen in HIV-infected in-patients, those initiating cART before admission were more likely to survive.
\end{abstract}

\section{INTRODUCTION}

The number of HIV-infected patients on combination antiretroviral therapy (cART) in middle and low income countries has rapidly increased and is projected to exceed 3.3 million by the end of 2008
(1, 2). In Kenya the National AIDS and STI Control Programme (NASCOP) initiated a free public antiretroviral treatment programme in 2005 and as of December 2007, 172,000 HIV-infected patients had initiated cART. However, close to 30,000 of these patients had either died or were lost to follow-up (3). 
In the developed world, where genotypic resistance testing and $3^{\text {rd }}$ line cART are standard of care, HIV disease computer simulations estimate the post-cART initiation life expectancy of a HIV-infected person to be between 24 and 36 years (4-6). Although cART is equally successfulin African patients (7-12) morbidity and mortality remain high $(13,14)$. Some of the reasons explicating this include: incomplete access to HIV-care; later start of antiretrovirals (CD4 cell count $<200 / \mathrm{ml}$ vs. $350 / \mathrm{ml}$ ); absence of $2^{\text {nd }}$ line regimes in some areas; and complete absence of genotyping and $3^{\text {rd }}$ line regimes. For these and other reasons, sub-Saharan Africa lags behind in HIV treatment and care provision and bears the brunt of HIV associated morbidity and mortality. Out of the 2 million HIV / AIDS related deaths worldwide in 2007, sub-Saharan Africa accounted for 1.6 million (15).

This prospective cohort study was undertaken at a public referral hospital in western Kenya where a free antiretroviral treatment programme has been in place since November 2001 (16). HIV infected patients enrolled in an ambulatory HIV care programme who got admitted to the adult medical wards were studied in order to determine their admissions diagnosis, length of stay in the ward, status and duration on cART and outcomes of their admission. We also sought to establish characteristics that specifically put HIV-infected patients at risk of dying despite having been enrolled in an antiretroviral programme.

\section{MATERIALS AND METHODS}

Design: The Moi University School of Medicine/ Moi Teaching and Referral Hospital Institutional Research and Ethics Committee (IREC) and Indiana University Purdue University at Indianapolis / Clarion Institutional Review Board (lRB) approved this study. Patient demographic and clinical data were collected prospectively from adult $\mathrm{HIV}$-infected patients admitted in the medical wards of the Moi Teaching and Referral Hospital (MTRH) between January 2005 and December 2006. Additional demographic, clinical and laboratory data were extracted from the AMPATH Medical Records System (AMRS), a fully electronic database of HIV-infected patients $(17,18)$.

Study site: MTRH, a national referral hospital in western Kenya, serves a population of 13 million people [ $(40 \%)$ of Kenya's population, national HIV prevalence of $6.7 \%(19,20)]$. MTRH also serves as the teaching hospital for Moi University School of Medicine (MUSoM). The total bed capacity at MTRH is 500 with 140 of these in two adult medical wards. Within this teaching hospital all inpatient management is overseen by physicians from the Department of Medicine of MUSoM. All ambulatory HIV-care in MTRH is provided by the Academic Model for Prevention and Treatment of HIV / AIDS (AMPATH) programme. The USAID-AMPATH Partnership (referred to as AMPATH hereafter) is a collaboration between MTRH, MUSoM and a consortium of universities from North America led by Indiana University School of Medicine and is funded in large part by USAID. The programme has been providing comprehensive ambulatory care to HIVinfected patients in western Kenya since November 2001. AMPATH provides an uninterrupted supply of antiretrovirals and medicines for treatment and prevention of opportunistic infections.

In the time period of this study AMPATH clinicians' involvement in care provision for admitted HIV-infected patients at MTRH included: clinical decision support with regard to antiretroviral initiation discontinuation and management of severe side effects; ensuring that antiretrovirals were uninterrupted during the admission; and supervision of a universal provider initiated HIV counselling and testing (PITC) programme for inpatients who had not been tested and / or enrolled in AMPATH.

Subjects: Analyses included all HIV-infected adult patients who got admitted to the adult medical wards at MTRH between January 2005 and December 2006 and had previously been enrolled in the AMPATH ambulatory care programme.

Data collection and management: Using a standardised form, a research assistant recorded, on a daily basis, the details of AMPATH patients admitted in the adult medical wards. Information collected included AMPATHidentification number, admission diagnosis, laboratory and radiological test results, treatment given, the length of stay on the ward and the final admission outcome (discharge or death). All data collected were entered into an admissions database programmed in MS Excel ${ }^{\circledR}$. In order to complete the patients' profiles, the AMPATHidentification number was used to link admissions data with pre-admission electronic data stored in the AMPATH Medical Record System (AMRS), a fully secure electronic medical database (http://amrs.iukenya.org). Thesedata included baseline data such as age, sex, employment status, marital status, weight, WHO stage and CD4 cell count closest but prior to admission and clinic appointment and medications adherence. All patient data were de-identified prior to analysis.

Data analysis: Events duration such as patient enrollment at AMPATH to admission, duration on CART and duration from admission to discharge or death were calculated. We independently determined the accuracy and likelihood of each diagnosis made by analysing patient history, physical examination results, progress notes and laboratory and radiological results available from hospital files of patients included in this study. For patients with multiple admissions, any subsequent diagnoses were excluded from this analysis if they were judged to be either unresolved or a continuation of initial diagnosis. All antiretroviral 
medications, adherence to antiretroviral and clinic appointment and lost to follow-up data wereabstracted from the AMPATH database. Loss to follow-up was defined as missing ambulatory clinic appointment for three consecutive months if on cART or six months if the patient had not initiated cART. Descriptive statistical analyses such as median and range were generated for continuous variables. Frequency tables were produced for categorical variables and these were compared via the Chi-square and Wilcoxon tests. Variables found to bestatistically significantat the $20 \%$ level wereincluded in a multivariatelogistic regression model to determine those that could be used independently to predict survival. Since we found that the majority of patients who died were not on cART treatment adherence data were not included in the final model since this data is collected for patients on cART only.

\section{RESULTS}

Between January 2005 and December 2006, 495 patients enrolled in AMPATH for ambulatory care contributed 524 admissions to the general adult medical wards atMTRH. The median age at admission was 38 years (range: $19-74$ years), $62 \%$ were female,
$55 \%$ were married, $90 \%$ had some formal education, $64 \%$ were employed and $375 / 495(76 \%)$ had initiated cART prior to admission. An additional $92(19 \%)$ patients initiated cART during admission. In total, $467 / 495(94 \%)$ of this cohort of patients were on cART. A total of 952 diagnoses were made with majority (58\%) of patients presenting with multiple complications. A diagnosis was not established in $16(2 \%)$ of the patients, $15(94 \%)$ of who died. Of all the diagnosis made (Table 1) tuberculosis was the commonest $(27 \%)$ complication followed by pneumonia (15\%) meningitis (11\%) diarrhoea (11\%), malaria $(6 \%)$, severe anaemia (4\%) and toxoplasmosis (3\%). Pulmonary tuberculosis accounted for 79\% of all the cases of tuberculosis. Of the 137 patients with a sputum smear result, $87(73 \%)$ were positive. Bronchopneumonia, pneumocystis pneumonia and lobar pneumonia accounted for 39\%, 36\% and $24 \%$ of all cases of pneumonia respectively. Excluding tuberculous meningitis, cryptococcal meningitis accounted for $65 \%$ of all cases of meningitis and $97 \%$ of cases of chronic meningitis. Other relatively common admission diagnoses included Kaposi's sarcoma, stroke, chronic liver disease and hepatitis. Details of the relatively frequency of these and other complications are provided in Table I.

Table 1

Admission diagnoses in 495 HIV-infected inpatients at the Moi Teaching and Referral Hospital in western Kenya

$\begin{array}{lll}\text { Diagnosis } & \text { In } 495 \text { admitted } & \text { In } 147 \text { patients } \\ & \text { patients }(n=952) & \text { that died }(n=232)\end{array}$

Tuberculosis

Pulmonary tuberculosis Sputum smear positive Sputum smear negative No sputum smear results Tuberculous meningitis Tuberculous adenitis Tuberculous pleural effusion Milliary tuberculosis Tuberculosis of the spine Tuberculous peritonitis Tuberculous pericarditis

Pneumonia

Bronchopneumonia

Pneumocystis pneumonia

Lobar pneumonia

Meningitis

Cryptococcal meningitis

Acute bacterial meningitis

$\begin{array}{ll}257(27 \%) & 63(27 \%) \\ 202 & 51 \\ 87 & 3 \\ 50 & 16 \\ 65 & 32 \\ 27 & 7 \\ 12 & 1 \\ 5 & - \\ 4 & 1 \\ 4 & 3 \\ 2 & - \\ 1 & - \\ 140(15 \%) & 30(13 \%) \\ 55 & 14 \\ 51 & 9 \\ 34 & 7 \\ 104(11 \%) & 33(14 \%) \\ 68 & 24 \\ 34 & 9\end{array}$




\begin{tabular}{|c|c|c|}
\hline Chronic (undetermined aetiology) & 2 & 1 \\
\hline Diarrhoea & $102(11 \%)$ & $22(9 \%)$ \\
\hline Dysentery & 3 & - \\
\hline Malaria & $57(6 \%)$ & $9(4 \%)$ \\
\hline $\begin{array}{l}\text { CNC disorders: Stroke, encephalitis, } \\
\text { encephalopathy, acute psychoses, } \\
\text { depression, dementia, paraparesis / plegia, } \\
\text { quadruparesis / plegia, peripheral neuropathy, } \\
\text { brain abscess, ataxia }\end{array}$ & $39(4 \%)$ & 4 \\
\hline Severe anaemia & $37(4 \%)$ & 4 \\
\hline $\begin{array}{l}\text { Infective: Salmonella typhi, cellulites, } \\
\text { otitis media PID, tetanus, HZ, } \\
\text { CMV retinitis, measles, UTI, } \\
\text { nephritis, cholecystitis, PUO }\end{array}$ & $32(3 \%)$ & 2 \\
\hline $\begin{array}{l}\text { Metabolic: DM, HTN, ARF, CRF, } \\
\text { CHF, acute asthma, hypoglycaemia, } \\
\text { shock, pancreatitis }\end{array}$ & $32(3 \%)$ & 7 \\
\hline Toxoplasmosis & $26(3 \%)$ & $11(5 \%)$ \\
\hline Malignancies: Kaposi's sarcoma, lymphoma & $22(2 \%)$ & $9(4 \%)$ \\
\hline chronic liver disease, jaundice, hepatitis & $20(2 \%)$ & 3 \\
\hline Candidiasis: oro-pharyngeal, oesophageal & $18(2 \%)$ & 2 \\
\hline Peptic ulcer disease (PUD) & $14(1.5 \%)$ & 1 \\
\hline Deep vein thrombosis (DVT) & $10(1 \%)$ & 2 \\
\hline $\begin{array}{l}\text { Haematological: bleeding, thrombocytopaenia, } \\
\text { splenomegaly, pancytopenia }\end{array}$ & $9(1 \%)$ & 3 \\
\hline Adverse drug reaction: SJS*, hepatitis & $7(11 \%)$ & 2 \\
\hline Nutritional: HIV wasting syndrome,pellagra & $6(1 \%)$ & 1 \\
\hline Others; Addison's disease, pulmonary embolism & $3(0.5 \%)$ & - \\
\hline Undetermined diagnosis & $16(2 \%)$ & $15(6 \%)$ \\
\hline
\end{tabular}

${ }^{*}$ More diagnoses than patients since some patients had multiple diagnosis or were admittered more than once.

$\mathrm{PID}=$ pelvic inflammatory disease; $\mathrm{HZ}=$ herpes zoster; $\mathrm{CMV}=$ cytomegalovirus: $\mathrm{UTI}=$ urinary tract infection; PUO = pyrexia of unknown origin; $\mathrm{DM}=$ diabetes mellitus; $\mathrm{HTN}=$ hypertension; $\mathrm{ARF}=$ acute renal failure; $\mathrm{CRF}=$ chronic renal failure; $\mathrm{CCF}=$ chronic heart failure; $\mathrm{SJS}=$ Stephen Johnson's Syndrome

Out of all the admissions, $348(70 \%)$ patients survived and $147(30 \%)$ died. The characteristics of patients who survived and those who died are shown in Table 2. Age, sex, marital and educational status were comparable between the two groups. There were no significant differences in WHO staging closest but prior to admission $(\mathrm{p}=0.2766)$. However, patients who died were more likely to be more severely immunosuppressed than those who survived ( $p=0.0059)$, had been enrolled in AMPATH more recently, (median $44 \mathrm{vs.} 70$ days; $\mathrm{p}=0.0248$ ) and were on antiretrovirals for a shorter duration of time (median 41 vs. 67 days; $p=0.0022$ ). Surviving patients were also more likely to have initiated antiretroviral treatment prior to admission $(\mathrm{p}<0.0006)$. The median duration of stay in the ward for surviving patients was eight days (range: 1 - 44 days) and six days (range: 1 - 30 days) for those who died $(\mathrm{p}=0.0024)$. Patients who died were also more likely to have been previously lost to follow-up from the ambulatory care clinic at AMPATH $(\mathrm{p} \leq 0.0001)$. For patients who died tuberculosis $(27 \%)$, meningitis $(14 \%)$, pneumonia $(13 \%)$, diarrhoea $(9 \%)$, severe anaemia $(6 \%)$ and toxoplasmosis $(5 \%)$ were the main complications diagnosed, which reflects a similar trend to the entire cohort of HIV-infected patients under study. In a multivariate logistic regression model that controlled for gender, cART status and duration, duration in AMPATH care, CD4 cell count and loss to follow-up, we found that patients were more likely to survive if they initiated cART prior to admission, (odds ratio 8; 95\%, C1 2-142). We also established 
that patients adherent to their clinic appointments prior to admission had markedly increased chances of surviving index admission compared to those lost to follow-up (odds ratio 6; 95\% CI 2 - 14). Gender and CD4 cell count were not significantly associated with admission's outcome for this specific cohort.

\section{DISCUSSION}

While it is of major concern that HIV-infected patients with access to comprehensive HIV care should record such high mortality rates, we would like to note that the majority of these deaths occurred soon after enrolment in AMPATH and initiation of cART, if at all. This mirrors our earlier findings that most deaths in patients initiating cART occurred within the first two months of treatment (21). This suggests that implementing a more aggressive clinic or home-based follow-up programme might reduce early mortality. It is also evident that the patients we studied were severely immunosuppressed, had multiple severe diseases at admission and hence at the greatest risk for developing life-threatening complications. Since it can be very challenging to diagnose some of the opportunistic infections implicated in resource poor settings, we recommend that clinicians have a high index of suspicion and a low threshold for diagnosing and aggressively treating these and other diseases in admitted HIV-infected patients.

It is not surprising that tuberculosis was common in this cohort since Kenya is listed as one of the ten countries with the highest burden of tuberculosis in the world (22). Previous precART studies on HIV-infected patients in Kenya have also shown a high prevalence of tuberculosis (23). However, the prevalence of tuberculosis in our patients was markedly lower $(27 \%)$ than that reported $(50 \%)$ in the study above. We speculate that this might be related to the fact that the majority of our patients were on CART. The finding of pulmonary tuberculosis as the commonest form of tuberculosis in these patients is consistent with that reported elsewhere (24). Since reactivation of latent tuberculous is common in HIV-infected patients (25), establishing tuberculosis screening and prevention programmes in the community might be an effective strategy to reduce the reservoir of Mycobacterium tuberculosis. Early recognition and diagnosis of HIV infection among community dwellers with subsequent administration of isoniazid preventive therapy (IPT) is another strategy that, if executed judiciously, has shown benefits in reducing tuberculosis reactivation in HlV-infected patients $(25,26)$.

It is likely that some of our subjects on cART had immune reconstitution and inflammatory syndrome (IRIS) which is being increasingly recognised as an early complication associated with successful immune recovery. IRIS, which represents a reaction to a previously immunologically unrecognised or indolent infection, may occur in up to $30 \%$ of patients initiating CART and is of particular concern in high tuberculosis prevalence areas $(27,28)$. IRIS has also been reported to occur in patients with cryptococcal meningitis. Pneumocystis pneumonia, Kaposi's sarcoma, herpes zoster and herpes simplex infections (27-29), most of which were common diseases in this study. Diagnosis of IRIS is particularly challenging in this setup because its presentation is very similar to traditional opportunistic infections and resources for laboratory, radiological and pathological diagnosis are not often at hand to be of assistance (30). However, the knowledge generated from such research studies as this one can guide clinicians in making empiric clinical diagnosis for some of these conditions.

Study limitations: Some of the diagnoses made were based on clinical and radiological investigations without bacteriologic, immunologic or postmortem proof. Since the correctness of clinical diagnosis is heavily dependent on clinicians' experience and judgment, and even though the investigators made efforts to verify each diagnosis, the accuracy of some of these diagnoses may vary. Other co-morbid conditions such as derangements in liver enzymes and electrolytes and their relative contribution to morbidity and mortality were not established. Similarly, the actual causes of death could not be determined since there were no postmortem data. Previous opportunistic infections, past admissions and their probable effect on current admission were not evaluated in this study. Due to financial constraints, plasma viralload tests are only performed on patients clinically failing cART, and hence were not included in this analysis.

In conclusion, the high death rates reported in this study occurred in a highly select cohort of HIVinfected inpatients, a few of them having recently initiated cART at an ambulatory HIV care programme in western Kenya. Most of these inpatients were severely immunosuppressed hence at the greatest risk acquiring life threatening complications such as tuberculosis, meningitis and pneumonia. On the contrary, patients that had been enrolled and initiated cART for longer duration were likely to survive. We would, therefore like to emphasise the need for early and accurate diagnosis of HIV, aggressive treatment of opportunistic infections in order to reduce morbidity and immortality and implementation of programmes for close follow-up early in the course of cART with interventions to increase adherence to treatment and clinic appointments. Future research should study the effects of such interventions on early morbidity and mortality in cART-treated HIV-infected adults.

\section{ACKNOWLEDGEMENTS}

We would like to specially appreciate the efforts of our research assistant, RachelNjeri (posthumous) who diligently collected admissions data presented in this study. We are thankful to Jepchirchir Kiplagat and 
Cecelia Chebet for data collation and transcription. We also recognise the efforts of all staff at the MTRH medical wards that provide care to HIV-infected patients under very constrained conditions. We are also grateful to all organisations that have input in the AMPATH programme including the Centres for Disease Control (CDC), Prevention of Maternal To Child Transmission - Plus (MTCT - Plus) Initiative, National AIDS and STI Control Programme (NASCOP), National AIDS Control Council (NACC) and the United States Agency for International Development President's Emergency Programme For AIDS Relief (USAID - PEPFAR).

\section{REFERENCES}

1. World Health Organisation. Towards Universal Access: Scaling Up Priority HIV / AIDS Interventions in the Health Sector. AProgress Report. Geneva:World Health Organisation; 2007.

2. Galarraga, O., O'Brien, M.E, Gutierrez, J.P., et al Forecast of demand for antiretroviral drugs in low and middle-income countries:2007-2008. AIDS. 2007; 21: S97 - S103.

3. National AIDS Control Council, Office of the President, Kenya. UNGASS 2008 Country Report for Kenya. NACC, Nairobi. 2008.

4. Lohse, N., Hansen, A.B., Gertoft, J., et al. Improved survival in HIV-infected persons: consequences and perspectives. J. Antimicrob. Chemother. 2007; 60: 461-463.

5. Lima, V.D., Hogg, R.S., Harrigan, P.R., etal. Continued improvement in survival among HIV-infected individuals with newer forms of highly active antiretroviral therapy. AIDS. 2007; 21: 685-692.

6. Schackman, H.R., Gebo, K.A., Walensky, R.P., et al. The lifetime cost or current human immunodeficiency virus care in the United States. Med. Care. 2006; 44:990-997.

7. Stringer J.S., Zulu, I., Levy, J., et al. Rapid scale-up of antiretroviral therapy at primary care sites in Zambia: feasibility and early outcomes. J. Amer. Med. Assoc. 2006; 296:782-793.

8. Wools-Kaloustian, K., Kimaiyo, S., Diero, L., C. et al. Viability and effectiveness of large scaleHIV treatment initiatives in sub-Saharan Africa: Experience from western Kenya. AIDS. 2006;20: 41-48.

9. Ivers, L.C., Kendrick, D. and Doucette, K. Efficacy of antiretroviral therapy programs in resource poor settings: a meta-analysis of the published literature. Clin. Infect. Dis. 2005; 41: 217-224.

10. Laurent, C., Ngom, G., N.F., Ndour, C.T., et al. Longterm benefits of highly active antiretroviral therapy in Senegalese HIV-1-infected adults. J. Acquir. Immune Defic. Syndr. 2005; 38: 14-17.

11. Coetzee, D., Hildebrand, K., Boulle, A., et al. Outcomes after two years of providing antiretroviral treatment in Khayelitsha, South Africa. AIDS. 2004; 18:887-895.

12. Weidle, P.J., Malamba, S., Mwebaze, R., et al. Assessment of a pilot antiretroviral drug therapy programme in Uganda: patients' response, survival. and drug resistance. Lancet. 2002; 360: 34-40.

13. Nyirenda, M., Hosegood, V., Barnighausen, T., et al. Mortality levels and trends by HIV serostatus in rural South Africa. AIDS 2007; 21: S73-S79.
14. Braitstein, P., Brinkhof, M.W., Dabis, F., et al. Mortality or HIV -1-infected patients in the first year of antiretroviral therapy: comparison between low income and high-income countries. Lancet. 2006; 367: 817-824.

15. UNAIDS: AIDS epidemic update: December 2007. "UNAIDS/07.27E/JC 1322E".

16. Einterz, R.M., Kimaiyo, S., Mengech, H.N.K., et al. Responding to the HIV pandemic: The power of an academic medical partnership. Acad. Med. 2007; 82:812-818

17. Tierney, W.M., Rotich, J.K., Hannan, T.J., et al. The AMPATH Medical Record System: Creating, implementing. and sustaining an electronic medical record system to support HIV / AIDS care in western Kenya. Medinfo. 2007; 12: 372-376.

18. Siika, A.M., Rotich, J.K.,Simiyu, C.J., et al. Anelectronic medical record system for ambulatory care of HIV -infected patients in Kenya. Int. J. Med. Informat. 2005; 74: 345-355.

19. Kenya National Census. Central Bureau of Statistics, Ministry of Planning and National Development. 1999.

20. The Kenya Demographic and Health Survey. Central Bureau of Statistics. Ministry of Planning and National Development. 2003

21. Siika, A.M., Wools-Kaloustian, K., Mwangi, A. W., et al. Risk factors for death in HIV-infected adult African patients receiving antiretroviral therapy (under review) East Afri. Med. J.

22. Global tuberculosis control: surveillance, planning, financing. WHO report 2007. Geneva. Organisation (WHO/HTM/TB/2007.376).

23. Rana, F.S., Hawken, F.P., Mwachari, P., et al. Autopsy study of HIV-1 positive and HIV -I negative adult medical patients in Nairobi, Kenya. J. Acquired Immune. Defic. Syndr. 2000; 24: 23-29.

24. Harries, A.D. Tuberculosis and human immunodeficiency virus infection in developing countries. Lancet. 1990; 335: 387-390.

25. Prevention and treatment of tuberculosis among patients infected with human immunodeficiency virus: principles of therapy and revised recommendations. Centres for Disease Control and Prevention. MMWR Recomm. Rep. 1998; 47(RR-20): $1-58$.

26. Golub, J.E., Saraceni, V., Cavalcante, S.C., et al. The impact of antiretroviral therapy and isoniazid preventive therapy on tuberculosis incidence in HIVinfected patients in Rio de Janeiro, Brazil. AIDS. 2007; 21: 1441-1448.

27. Shelburne, S.A., Visnegarwala, F., Darcourt, J., et al. Incidence and risk factors for immune reconstitution inflammatory syndrome during highly active antiretroviral therapy. AIDS 2005; 19: 399-406.

28. Murdoch, D.M., Venter, W.D., Feldman, C., et al. Incidence and risk factors for the reconstitution inflammatory syndrome in HIV patients in South Africa: a prospective study. AIDS. 2008; 22: 601-610.

29. Bower, M., Nelson, M., Young, A.M., et al. Immune reconstitution inflammatory syndrome associated with Kaposi's sarcoma. J. Clin. Oncol. 2005; 23: 52245228.

30. Colebunders, R., John, L. and Huyst, V. Tuberculosis immune reconstitution inflammatory syndrome in countries with limited resources, Int. J. Tuber. Lung. Dis. 2006; 10: 946-953. 